\title{
FORUM
}

\section{Neue Wege bei den EU-Eigenmitteln gehen}

\author{
Helga Trüpel und Jan Seifert*
}

Mittlerweile hat man sich schon daran gewöhnt. Jeder große EU-Kompromiss, der im Einstimmigkeitsverfahren ausgehandelt wird, impliziert bereits seine eigene Überarbeitung. Das war mit dem Nizza-Vertrag so, und auch mit der Finanziellen Vorausschau 2007-2013 verhält es sich nicht anders. Auf ihrem Gipfel im Dezember 2005 vereinbarten die Staatsund Regierungschefs, dass die Kommission bereits 2008/9 eine Überprüfung (mid-term review) vornehmen solle. Dieser Bericht wird ausdrücklich die Einnahmen- wie auch die Ausgabenseite umfassen. Sollte es im Zuge dieser Reformüberlegungen nicht gelingen, zu einem neuen Haushaltsmechanismus zu kommen, werden sich die Regierungen spätestens 2011 ,an die Gurgel“ gehen, wie Bundeskanzler Schüssel prophezeite. ${ }^{1}$

Die Zeit bis zur Überprüfung und den folgenden Europawahlen im Juni 2009 sollte nun genutzt werden, um unter Einbeziehung aller Ebenen neue Wege einer qualitativen EUHaushaltsreform zu diskutieren. Dieser Beitrag soll dazu mit besonderem Blick auf die Eigenmittel der EU einige Vorüberlegungen liefern.

\section{Das System der EU-Eigenmittel}

Die EU besitzt derzeit drei Typen von Eigenmitteln, die sie auf Grundlage von Artikel 269 EGV bei den Mitgliedstaaten abschöpft.

\section{Tabelle 1: Typen von Eigenmitteln}

\begin{tabular}{|l|l|l|l|}
\hline & Quelle & Anteil 1992 (EU-12) & Anteil 2004 (EU-25) \\
\hline 1. Säule & Traditional Own Resources & $24 \%$ & $13 \%$ \\
\hline 2. Säule & Mehrwertsteueranteil & $62 \%$ & $14 \%$ \\
\hline 3. Säule & Bruttonationaleinkommen & $14 \%$ & $73 \%$ \\
\hline & Gesamtvolumen & $€ 60 \mathrm{Mrd}$ & $€ 104 \mathrm{Mrd}$ \\
\hline
\end{tabular}

Als traditionelle Eigenmittel (,Traditional Own Resources', TOR) gelten die Zoll- und Agrarabgaben, die auf Grundlage der EU-Kompetenz im Agrar- und Außenhandelsbereich erhoben und an die EU abgeführt werden. An zweiter Stelle kommen seit 1980 die Mehrwertsteueranteile hinzu, die in einer komplexen Formel in Abhängigkeit vom Bruttonationaleinkommen (BNE) berechnet werden und derzeit vereinfacht etwa 0,3 Prozent der Mehrwertsteuerberechnungsgrundlage bilden. Als dritte Säule verbleiben nationale Haushaltszuschüsse, die auf Grundlage des BNE, das heißt der Wirtschaftskraft der einzelnen

* Dr. Helga Trüpel, MdEP, Mitglied des Haushaltsausschusses des Europäischen Parlaments.

Jan Seifert, wissenschaftlicher Mitarbeiter von Dr. Helga Trüpel, MdEP.

1 Vgl. Süddeutsche Zeitung vom 31.12.2005. 
Mitgliedstaaten, berechnet werden. Anzumerken ist mit Blick auf die dritte Säule, dass sie den ,Britenrabatt' umfasst, der wiederum mit der neuen Finanziellen Vorausschau um Ausgleichsrabatte für weitere ,Nettozahler' ergänzt wird (Niederlande, Deutschland, Schweden, Österreich).

Mit seinem eindeutigen Schwerpunkt auf nationalen Haushaltsbeiträgen, die abhängig von der Wirtschaftsleistung (BNE, angepasste Mehrwertsteuer) sind, ist das Beitragssystem damit proportional zum Wohlstandsniveau der Mitgliedstaaten und nicht wie die meisten nationalen Steuersysteme progressiv. Darüber hinaus spiegelt diese Art der Finanzierung (mit Ausnahme der traditionellen Eigenmittel) die ,Clubidee' einer internationalen Organisation wie der UNO wider, in der die Bürger der Mitgliedstaaten ausschließlich durch ihre Regierungen repräsentiert werden. Eine solche Finanzierung ignoriert, dass die EU keine internationale Organisation ist. Die Union ergänzt den Föderalismus aus kommunaler, Länderund Bundesebene um die kontinentale Ebene und tritt mit ihren spezifischen Aufgaben neben die Mitgliedstaaten.

\section{Einigkeit über die Reformnotwendigkeit}

Nicht nur Bundeskanzlerin Merkel, Bundeskanzler Schüssel und Kommissionspräsident Barroso haben beim Dezembergipfel die Reformnotwendigkeit des Eigenmittelsystems anerkannt. Auch die europäischen und nationalen Parlamentarier waren sich auf dem Interparlamentarischen Forum am 8. und 9. Mai 2006 darüber einig. Ausgangspunkt für die anstehenden Reformüberlegungen sind vier Prämissen:

- Das aktuelle System ist nicht mehr tragbar, weil es als zu komplex, unfair und intransparent angesehen wird.

- Die Reform der Einnahmen und Ausgaben hängen zusammen.

- Alle Rabatte gehören auf den Verhandlungstisch.

- Eine zusätzliche EU-Steuer (etwa in Form einer direkten Einkommenssteuer) wird abgelehnt.

Wie diese Vorgaben im Einzelnen angegangen werden sollten wird unter den Abgeordneten noch diskutiert. Um eine Frage allerdings kreisen alle Argumente zumindest aus Perspektive der ,Nettozahlerländer', und das ist die Frage der Ausgabenakzeptanz. So lange der Eindruck besteht, dass bestimmte Gruppen beziehungsweise Regionen überproportional von EU-Mitteln profitieren, wird sich kaum eine Unterstützung für Reformen herbeiführen lassen, die zwangsläufig auf irgendeiner Seite zu Mehrbelastungen und damit zu möglichen Vetos führen wird. Bevor potenzielle Lösungswege diskutiert werden, muss die Widersprüchlichkeit des Haushalts durch einen Blick in die Ausgabenstruktur noch einmal vor Augen geführt werden.

\section{Keine Eigenmittelreform ohne Ausgabenreform}

Trotz aller Modernisierungsrhetorik weist auch die neue Finanzielle Vorausschau rund 42 Prozent der Ausgaben im Agrarbereich und circa 36 Prozent für die Strukturfonds aus. Nur rund 8 Prozent gehen in die Rubrik 1a „Wettbewerbsfähigkeit“ und damit in vorrangig wachstums- und wissensorientierte Bereiche. ${ }^{2}$ Verglichen mit dem Kompromiss der Regierungschefs vom Dezember 2005 ist das zwar insgesamt ein Trippelschritt nach vorne, stellt aber weiterhin eine zutiefst strukturkonservative Ausgabenstruktur dar. Weil niemand von

2 Vgl. Peter Becker: Fortschreibung des Status Quo - Die EU und ihr neuer Finanzrahmen Agenda 2007, in: integration 2/2006, S. 106-121. 
seinem Veto abrücken will und der Haushalt damit weiterhin eher der Zusammenfassung von 25 ,Ersatzhaushalten“ gleicht (80 Prozent der Mittel werden direkt von den Mitgliedstaaten ausgegeben), wird kaum eine Wachstumsstimulierung zu erwarten sein. Weiterhin unverständlich bleiben strukturelle Widersprüche, die sich über den gesamten Haushalt verteilen und für weitere sieben Jahre manifestiert sind.

Erstens: Keiner konnte bisher plausibel argumentieren, warum die Gemeinsame Agrarpolitik (GAP) die einzige Politik bleibt, die keine Kofinanzierung erfordert. Mit einer Umstellung auf nationale Kofinanzierung von 25 Prozent würde allein der Haushalt 2006 um rund 10 Milliarden Euro verringert werden. Dies ist bei weitem der umfangreichste und meistdiskutierte Ansatz zur Ausgabenoptimierung.

Zweitens: Weil mit der angestrebten Vollendung des Binnenmarktes seit den 1980ern wirtschaftliche und soziale Veränderungen insbesondere in der Peripherie erwartet wurden, schuf man die Strukturfonds, um Disparitäten in den betroffenen Regionen abzubauen. Schon seit längerem sind die Strukturfonds zur zweiten Säule nationaler Infrastrukturpolitik geworden, deren Verteilung mehr denn je das Ergebnis von Verhandlungen ist, anstatt auf klaren und einheitlichen Kriterien den Menschen in den abgehängten Regionen über eine absehbare Periode gezielte Förderung zu gewähren. Aus diesem Grund brauchen wir eine Neuausrichtung der Strukturfonds an Hand qualitativer Kriterien. Der neue Förderschwerpunkt auf innovativen Ideen und Arbeitsplätzen ist richtig und muss entsprechend umgesetzt werden. Idealerweise würden die Kohäsionsfonds im Zuge der Reformüberlegungen in diese neuen Strukturen überführt werden.

Drittens: Wenn auch sehr viel begrenzter im Umfang, so stehen für viele die Subventionen für den Tabakanbau (circa 900 Millionen Euro pro Jahr) oder der Reisezirkus des Europäischen Parlaments (rund 200 Millionen Euro pro Jahr) genauso exemplarisch für Lobbyismus und die Widersprüchlichkeit des europäischen Haushalts wie die Agrar- und Strukturpolitik.

\section{Kriterien für eine Eigenmittelreform}

Allen Beteiligten dürfte klar sein, dass eine über Jahrzehnte gewachsene Ausgabenstruktur nicht einfach zu verändern ist. Zu bedauern ist, dass sich auch seit dem ,Schreyer-Bericht' von $2004^{3}$, nichts Konkretes in Richtung einer Eigenmittelreform getan hat. Für eine grundlegende Reform sollten unserer Meinung nach folgende Bewertungskriterien Beachtung finden:

Akzeptanz: Jede staatliche Ausgabe wird letztlich aus den Steuermitteln der Bürger und Unternehmen bezahlt. Aus diesem Grund muss durch eine zukunftsfähige Ausgabenstruktur in erster Linie die Akzeptanz dafür hergestellt werden, dass spezifische Ausgaben auf EUEbene vorzunehmen sind.

Transparenz und Einfachheit: Gerade um das erste Ziel der Akzeptanz zu erreichen, müssen die Einnahmen und Ausgaben der Union transparent und einfach zu verstehen sein. 80 Prozent der Mittel werden von den Mitgliedstaaten verwaltet und ausgegeben, und diese wiederum lassen sich in den meisten Fällen nicht in die Karten schauen, bei wem das Geld am Ende ankommt. ${ }^{4}$ Auf der anderen Seite versteht die EU-Normalbürgerin genauso wenig wie ihr Finanzminister die Berechnung der Mehrwertsteueranteile. Diese Komplexität sät Misstrauen, was wiederum dazu führt, dass jeder sich durch die andere(n) Seite(n) übervorteilt sieht.

3 Vgl. Europäische Kommission: Finanzierung der Europäischen Union - Bericht der Kommission über das Funktionieren des Eigenmittelsystems, KOM(2004)0505 endg.

4 Vgl. Agrarausgaben durch die deutschen Bundesländer. Für einen Überblick der Verteilung von Agrarausgaben in den EU-Mitgliedstaaten siehe: www.farmsubsidy.org (letzter Zugriff: 31.05.2006). 
Gerechtigkeit: In den Verträgen liest man, dass die Europäische Union sich zum Wert der Solidarität bekennt und die Konvergenz ihrer Volkswirtschaften anstrebt. Daraus ließe sich ableiten, dass das Geld zweckgebunden, mehrwertig und zur Unterstützung der sozialen Kohäsion verwendet wird.

Doppelcharakter der Union: Genauso wie die Union ihre verfassungsgemäßen Kompetenzen von den Mitgliedstaaten und Bürgern ableitet, sollten auch die Eigenmittel diesen Doppelcharakter der Union reflektieren, indem neben Beiträgen aus den Mitgliedstaaten auch direkte Beiträge der Bürger stehen. Dies erhöht die Legitimität der Ausgaben und verstärkt die Verantwortung von Parlament und Rat für eine sinnvolle Ausgabenpolitik.

Konnexitätsprinzip: Aufgaben, die an die EU delegiert werden, weil sie von ihr besser ausgeübt werden können, müssen auch ausreichend finanziert sein. Dies ist zwar technisch über die ad-hoc Bereitstellung von Krisenmitteln und Flexibilitätsinstrumenten möglich, kann allerdings keine nachhaltige Lösung sein. Umgekehrt gilt genauso, dass EU-Mittel ausschließlich für europäische Aufgaben verwendet werden sollten.

Ausgabenneutralität: Ausgangspunkt einer großen Eigenmittelreform muss sein, dass unter dem Strich keine steuerliche Mehrbelastung steht. Einer Anhebung von jederlei Abgabe müsste immer die entsprechende Verringerung des BNE-Anteils gegenüberstehen. Anders ließe sich eine solche Reform schwer legitimieren. Gleichzeitig müsste im Zuge der Reformüberlegungen mittelfristig hinterfragt werden, ob die entsprechenden Politiken - besonders im Feld der Außen- und Sicherheitspolitik ${ }^{5}$ - unter den gegebenen Umständen ausreichend finanziert sind.

\section{Wege zur Eigenmittelreform}

Nach dem Gesprächsbeginn am 8. und 9. Mai auf dem Interparlamentarischen Forum war zunächst die gemeinsame Sitzung nationaler und europäischer Abgeordneter in Brüssel abzuwarten, bevor der Berichterstatter des Europäischen Parlaments, Herr Lamassoure, seinen Bericht im Herbst vorlegt. Dieser sollte als erste europäische Stellungnahme eine breitere Diskussion auslösen. Eine permanente Arbeitsgruppe nationaler und europäischer Abgeordneter sollte die Fragen dann aufgreifen und gemeinsam beginnen, Lösungswege auszuarbeiten, die in das Weißbuch der Kommission 2008 einfließen können.

Eines ist aber klar. Wer nicht nur marginale Korrekturen an den Berechnungsformeln des Mehrwertsteueranteils oder des Britenrabatts vornehmen will, der muss auch akzeptieren, dass alle Einnahmen- und Ausgabenposten auf den Verhandlungstisch gehören. Hierzu zählen an vorderster Stelle der Britenrabatt und die Gemeinsame Agrarpolitik.

\section{Verhandlungsstrategien}

Um einen unlösbaren britisch-französischen Schlagabtausch zwischen Britenrabatt und GAP zu vermeiden, muss die Gesamtstruktur des Haushalts in den Vordergrund gestellt werden. Natürlich werden die Salden und Rabatte der Mitgliedstaaten eine zentrale Rolle spielen. Wer aber die europäischen Bürger überzeugen will, muss nicht nur zu einer Ausgabenstruktur kommen, die die Prioritäten derselben widerspiegelt, sondern den Bürgern auch ihren Mehrwert vorrechnet und aus Bürgersicht für jeden einzelnen gerecht erscheint. Vielleicht lohnt es sich dafür, einmal die nationale ,Haushälterperspektive` zu verlassen.

Da es sich nicht vermeiden lässt, dass die mitgliedstaatlichen Regierungen an zentraler Stelle an den Verhandlungen beteiligt sind, fassen wir die Kernpunkte der einzelnen Interes-

5 Eine gemeinsame Sicherheitspolitik würde eine Ausgabenverlagerung auf die europäische Ebene bedeuten, könnte und sollte aber durch bessere Kooperation auch zu Einsparungen und Effizienzsteigerungen führen. 
sen kurz zusammen (siehe Tabelle 2). Offensichtlich gibt es keinen Einstiegspunkt in die Verhandlungen, bei dem alle Akteure übereinstimmen. Jeder hat im EU-Haushalt beziehungsweise in der Finanzierung seinen eigenen Schwerpunkt unterbringen können. Darum sollte es am vielversprechendsten sein, dort anzusetzen, wo einzelne Mitgliedstaaten über Einzelinteressen wachen. Dies ist der Fall beim Britenrabatt, aber auch bei der in den letzten Wochen entfachten symbolischen Debatte über den zweiten Sitz des Parlaments in Straßburg.

Tabelle 2: Interessen von Schlüsselakteuren bei der Eigenmittelreform

\begin{tabular}{|c|c|c|}
\hline Mitgliedstaat & Situation & Interesse \\
\hline Belgien & $\begin{array}{l}\text { überproportional hohe Zolleinnahmen, } \\
\text { Sitz zahlreicher Institutionen: } 57 \% \text { der } \\
\text { administrativen EU-Ausgaben (2004) }\end{array}$ & Beibehaltung der $25 \%$ Zollanteile \\
\hline Deutschland & $\begin{array}{l}\text { Größter absoluter Nettozahler, Unterstüt- } \\
\text { zung für derzeitige GAP in einflussrei- } \\
\text { chen Bundesländern, kritische Lage in } \\
\text { Ostdeutschland und Grenzregionen, Be- } \\
\text { fürchtungen: verschärfter Wettbewerb bei } \\
\text { Unternehmenssteuern }\end{array}$ & $\begin{array}{l}\text { Abschaffung Britenrabatt, Reform } \\
\text { der Haushaltsstruktur (weniger } \\
\text { GAP, mehr GASP), Fortführung } \\
\text { Förderung für Ostdeutschland und } \\
\text { östliche Grenzregionen, kann von } \\
\text { stärkerer Rubrik 1a profitieren }\end{array}$ \\
\hline Frankreich & $\begin{array}{l}\text { Nettozahler, mit Abstand größter Profi- } \\
\text { teur der GAP, Befürchtungen: verschärf- } \\
\text { ter Wettbewerb bei Unternehmenssteuern }\end{array}$ & $\begin{array}{l}\text { Beibehalten der voll finanzierten } \\
\text { GAP, Abschaffung des Britenra- } \\
\text { batts, Festhalten an Straßburg als } \\
\text { Sitz des EP }\end{array}$ \\
\hline $\begin{array}{l}\text { zahlreiche mittel- und } \\
\text { osteuropäische Staaten }\end{array}$ & $\begin{array}{l}\text { Empfänger von Strukturmitteln, starke } \\
\text { Unterstützung ländlicher Regionen durch } \\
\text { GAP, niedrige Unternehmenssteuern }\end{array}$ & $\begin{array}{l}\text { ausreichende Finanzierung Struk- } \\
\text { turmittel für ärmste Regionen, Fort- } \\
\text { schreibung der GAP, Abschaffung } \\
\text { Britenrabatt, Steuerwettbewerb }\end{array}$ \\
\hline Niederlande & $\begin{array}{l}\text { Nettozahler, spätestens seit Referendum } \\
\text { besondere Aufmerksamkeit auf Beitrag, } \\
\text { überproportional hohe Zolleinnahmen }\end{array}$ & $\begin{array}{l}\text { Beibehaltung der 25\% Zollan- } \\
\text { teile, geringer Beitrag, Abschaf- } \\
\text { fung Britenrabatt oder Beibehal- } \\
\text { tung des Abschlags auf die } \\
\text { Finanzierung des Rabatts, gegen } \\
\text { Straßburg als Sitz des EP }\end{array}$ \\
\hline Spanien & $\begin{array}{l}\text { Mit } 28 \% \text { größter Empfänger von Struk- } \\
\text { turfonds (2004) }\end{array}$ & $\begin{array}{l}\text { Beibehaltung Strukturförderung } \\
\text { für nicht-ärmste Regionen }\end{array}$ \\
\hline $\begin{array}{l}\text { Vereinigtes } \\
\text { Königreich }\end{array}$ & $\begin{array}{l}\text { minimaler Nettozahler, 5,7 Mrd Euro Ra- } \\
\text { batt (2006) }\end{array}$ & $\begin{array}{l}\text { Beibehaltung niedriger Beitrag, } \\
\text { Reduzierung GAP, kann von stär- } \\
\text { kerer Rubrik 1a profitieren }\end{array}$ \\
\hline
\end{tabular}

\section{Neue Eigenmittelmodelle}

Obwohl es nicht an interessanten Ideen zu neuen (internationalen) Steuer- und Abgabenquellen mangelt gehen wir in der folgenden Diskussion auf Modelle ein, die innerhalb der bestehenden Strukturen und bis 2013 realisiert werden könnten. Diese umfassen neben Reformen der bestehenden Typen auch neue Quellen. Die folgende Tabelle 3 gibt einen Überblick über potenzielle Eigenmittelquellen, die zwar meist durch die Mitgliedstaaten erhoben, aber trotzdem originär in den EU-Haushalt fließen könnten. 
Tabelle 3: Potenzielle Eigenmittelquellen

\begin{tabular}{|c|c|c|c|}
\hline Quelle & Implementierung & Effekte & Aufkommen \\
\hline $\begin{array}{l}\text { Zölle und } \\
\text { Agrarabgaben }\end{array}$ & $\begin{array}{l}\text { gemeinsame Berechnungs- } \\
\text { grundlage besteht }\end{array}$ & $\begin{array}{l}25 \% \text { verbleiben derzeit auf } \\
\text { nationaler Ebene, Aufkom- } \\
\text { men wird wegen zunehmen- } \\
\text { der Handelsliberalisierung } \\
\text { weiter abnehmen }\end{array}$ & maximum $15 \%$ \\
\hline $\begin{array}{l}\text { Mehrwert- } \\
\text { steueranteil }\end{array}$ & $\begin{array}{l}\text { gemeinsame Berechnungs- } \\
\text { grundlage besteht, nicht } \\
\text { ausgewiesen für die Bürger }\end{array}$ & derzeit an BNE gekoppelt & $\begin{array}{l}\text { rund } 50 \% \text { bei ers- } \\
\text { tem } 1 \% \text { der Mehr- } \\
\text { wertsteuer }\end{array}$ \\
\hline BNE-Anteil & $\begin{array}{l}\text { gemeinsame Berechnungs- } \\
\text { grundlage besteht, kompli- } \\
\text { ziertes Berechnungsverfah- } \\
\text { ren }\end{array}$ & $\begin{array}{l}\text { brauchbar als ,Lückenfül- } \\
\text { ler‘, verstärkt Saldodebatte }\end{array}$ & variabel \\
\hline Flugmaut & $\begin{array}{l}\text { Daten werden schon über } \\
\text { Eurocontrol erhoben und } \\
\text { müssten mit Airlines an zen- } \\
\text { traler Stelle verrechnet wer- } \\
\text { den, implementierbar inner- } \\
\text { halb von 3-6 Jahren }\end{array}$ & $\begin{array}{l}\text { würde Flugverkehr über Eu- } \\
\text { ropa belasten, ökologischer } \\
\text { Lenkungseffekt, kann unab- } \\
\text { hängig von IATA Ver- } \\
\text { pflichtungen eingeführt } \\
\text { werden }\end{array}$ & $5-10 \%$ \\
\hline Kerosinsteuer & $\begin{array}{l}\text { Erhebung müsste (national) } \\
\text { neu aufgebaut werden, imp- } \\
\text { lementierbar innerhalb von } \\
\text { 3-6 Jahren }\end{array}$ & $\begin{array}{l}\text { Ende der Steuerfreiheit von } \\
\text { Flugbenzin, Änderung in } \\
\text { IATA Position müsste her- } \\
\text { beigeführt werden, teilweise } \\
\text { Wettbewerbsnachteil für eu- } \\
\text { ropäische Airlines }\end{array}$ & $\begin{array}{l}\text { Variabel, vielleicht } \\
5-10 \%\end{array}$ \\
\hline $\begin{array}{l}\text { Mineralöl- } \\
\text { steueranteil }\end{array}$ & $\begin{array}{l}\text { gemeinsame Berechnungs- } \\
\text { grundlage besteht, Erhe- } \\
\text { bung und Berechnung ist } \\
\text { mit einheitlicher Bemes- } \\
\text { sungsgrundlage nun mög- } \\
\text { lich, implementierbar in 3-6 } \\
\text { Jahren }\end{array}$ & $\begin{array}{l}\text { Verschiebungen anderer } \\
\text { Beiträge zu Ungunsten der } \\
\text { Nettozahler, falls Lenkungs- } \\
\text { effekt beabsichtigt (und da- } \\
\text { mit Erhöhung), trifft es vor } \\
\text { allem neue schwächere Mit- } \\
\text { gliedstaaten }\end{array}$ & $\begin{array}{l}\text { Rund } 50 \% \text { bei } \\
\text { Hälfte der Bemes- } \\
\text { sungsgrundlage }\end{array}$ \\
\hline $\begin{array}{l}\text { Unternehmens- } \\
\text { steueranteil }\end{array}$ & $\begin{array}{l}\text { setzt gemeinsame Bemes- } \\
\text { sungsgrundlage voraus, bis- } \\
\text { her umstritten }\end{array}$ & $\begin{array}{l}\text { weniger Steuermissbrauch } \\
\text { durch Konzerne, Transpa- } \\
\text { renz für Standortentschei- } \\
\text { dungen, potenziell Steuerer- } \\
\text { höhungen in einigen } \\
\text { Mitgliedstaaten }\end{array}$ & $\begin{array}{l}1 \% \text { würde } 100 \% \\
\text { finanzieren }\end{array}$ \\
\hline $\begin{array}{l}\text { Einkommens- } \\
\text { steueranteil }\end{array}$ & $\begin{array}{l}\text { setzt gemeinsame Bemes- } \\
\text { sungsgrundlage und Abfüh- } \\
\text { rungsmechanismen voraus, } \\
\text { sehr umstritten }\end{array}$ & $\begin{array}{l}\text { direkte Besteuerung der } \\
\text { Bürger, Gefahr von Steuer- } \\
\text { erhöhung }\end{array}$ & $\begin{array}{l}\text { variabel, bis zu } \\
100 \% \text { und mehr }\end{array}$ \\
\hline
\end{tabular}




\begin{tabular}{|l|l|l|l|}
\hline Quelle & Implementierung & Effekte & Aufkommen \\
\hline Emissionszertifikate & $\begin{array}{l}\text { werden nach EU-einheitli- } \\
\text { chen Regeln über nationale } \\
\text { Allokationspläne alle paar } \\
\text { Jahre verteilt (derzeit für } \\
\text { 2008-12), bis zu 10\% dürfen } \\
\text { versteigert/verkauft werden }\end{array}$ & $\begin{array}{l}\text { ökologischer Lenkungsef- } \\
\text { fekt, potenziell minimale } \\
\text { preise }\end{array}$ & $\begin{array}{l}\text { 2 Milliarden Euro }{ }^{1} \\
\text { (einmalig vor 2008) } \\
\text { für vier Jahre. } \\
\text { Keine Einnahmensi- } \\
\text { cherheit }\end{array}$ \\
\hline
\end{tabular}

Ausgehend von diesen neun Quellen wollen wir drei Modelle diskutieren, die auch stellvertretend für Beiträge der letzten Monate stehen und Chancen auf Umsetzung hätten. Diese drei Modelle, die wir hier ,Bundestag', ,Europamehrwert' und ,Ressourcenverbrauch" nennen, fassen die vorgestellten neun Optionen in Cluster zusammen und stehen gleichzeitig für drei sehr verschiedene Ansätze der EU-Finanzierung.

\section{Modell ,Bundestag“}

In der Anhörung des EP-Berichterstatters Lamassoure durch mehrere Bundestagsausschüsse am 10. Mai 2006 zeigte sich vor allem unter den großen Fraktionen eine breite Unterstützung für den Ausbau des BNE-Anteils. ${ }^{6}$ Der fraktionsübergreifende Kritikpunkt war die Fortführung des Britenrabatts. Zur weiteren Entlastung des EU-Haushalts wurde außerdem der Einstieg in die Kofinanzierung der GAP gefordert. Spielt man ein solches Modell mit 25 Prozent Kofinanzierung und dem Wegfall des Mehrwertsteueranteils einmal ausgehend vom Haushalt 2006 durch, ergibt sich unser Modell ,Bundestag', das freilich nicht auf einer formellen Beschlusslage basiert.

Im Ergebnis bleibt festzuhalten, dass durch den Abbau des massiven Britenrabatts (etwa 5,7 Milliarden Euro) und die Einführung der Kofinanzierung (etwa 10 Milliarden Euro) insgesamt Umschichtungen von rund 15 Milliarden Euro entstehen. Dies führt dazu, dass abgesehen von Großbritannien alle Mitgliedstaaten weniger zahlen, wobei freiwerdende Mittel auf nationaler Ebene potenziell zur Gegenfinanzierung der Agrarpolitik einzukalkulieren sind. Inwiefern sich die Briten darauf einlassen, ist aber zweifelhaft, zumal sie in diesem Fall weitere Mittel zur Agrar-Kofinanzierung aufbringen müssten. Der schwerwiegendere Nachteil dieses Modells ist aber, dass es an der juste-retour-Logik nichts ändern wird, da der Haushalt nun quasi ausschließlich aus direkten nationalen Beiträgen gespeist wird.

Dem Modell ,Bundestag ' wollen wir zwei originär europäische Modelle gegenüberstellen, die neben den Mitgliedstaaten auch die Bürger direkt in die Finanzierung der Union einbeziehen beziehungsweise ökologische Lenkungseffekte entfalten können.

\section{Modell ,Europamehrwert}

Innerhalb unseres Modells ,Europamehrwert‘ geht es darum, die zweite Säule der Finanzierung durch die Abführung eines ,Europamehrwerts‘ transparenter zu gestalten. Zentrales Element ist die Weiterleitung des ersten Prozentpunkts der Mehrwertsteuer direkt in den EUHaushalt. Daneben wird der Anteil der Zoll- und Agrarmittel, die den abschöpfenden Mitgliedstaaten verbleiben, wieder auf das alte Niveau von 10 Prozent zurückgeführt. Fehlende

6 Vgl. http://www.bundestag.de/aktuell/hib/2006/2006_140/06.html (letzter Zugriff: 12.06.2006). 
Haushaltsmittel werden durch einen BNE-Anteil ergänzt, der keine Rabatte mehr kennt. Auf der Ausgabenseite wird eine Kofinanzierung der Landwirtschaft von 25 Prozent eingeführt.

Die Direktausweisung von 1 Prozent der Mehrwertsteuer in Verbindung mit den anderen Schritten hat zur Folge, dass bis auf Großbritannien sowie in sehr geringen Margen Malta und Zypern alle Mitgliedstaaten weniger zahlen als vorher. Der Vorteil dieses Modells ergibt sich damit auf zwei Ebenen. Durch die direkte Verbindung der Konsumausgaben der Bürger mit der Finanzierung der EU wird nicht nur die Transparenz der ,Kosten der EU` erhöht sondern auch der Verantwortungsdruck europäischer Ausgabenpolitik gegenüber den Steuerzahlern gesteigert. Der zweite Aspekt dieses Modells ist die Absenkung der direkten nationalen Beiträge (nach BNE) von rund 80 Milliarden auf 30 Milliarden Euro. Durch die direkte Ausweisung (und Abführung) des Mehrwertsteuerpunkts zusammen mit dem leichten Anstieg der TOR, die von den Beteiligten quasi direkt an die EU abgeführt werden, ergibt sich eine wesentliche Entlastung der nationalen BNE-Beiträge. Ausgangsprämisse unserer Überlegungen zu diesem Modell ist allerdings die Aufkommensneutralität. Beim ,Europamehrwert' geht es darum, das eine Prozent der Mehrwertsteuer auf jeder Rechnung auszuweisen, um den Bürger-EU-Bezug herzustellen. Es darf nicht zu einer Steuererhöhung missbraucht werden.

\section{Modell ,Ressourcenverbrauch“}

Unser zweiter Vorschlag beinhaltet eine Erweiterung der ersten Säule um ökologische Steuern bei gleichzeitiger Streichung des Mehrwertsteueranteils. Die neuen Elemente bestehen aus der in der Übersicht beschriebenen Einführung einer Flugmaut, der Versteigerung von CO2-Emissionszertifikaten sowie der Abführung von 50 Prozent der gemeinsamen Bemessungsgrundlage für Mineralölsteuern. Wie auch im vorigen Modell wird der mitgliedstaatliche Anteil der Zoll- und Agrarmittel auf sein altes Niveau von 10 Prozent zurückgeführt. Fehlende Mittel werden durch den rabattfreien BNE-Anteil ergänzt. Zusätzlich wird die Landwirtschaft mit 25 Prozent kofinanziert.

Auch durch das Modell Ressourcenverbrauch ergeben sich massive Entlastungen bei den BNE-Direktzahlungen der Mitgliedstaaten (beispielsweise 14,6 Milliarden Euro für Deutschland). Die Mehrheit der Eigenmittel würde hier direkt über die (Energie-)Verbraucher erhoben werden ${ }^{7}$. Das Modell beinhaltet damit eine Abgabensteigerung für Ressourcenverbraucher und ist somit nicht, zielgruppenspezifisch“ aufkommensneutral, weil die entstehenden Entlastungen der nationalen Haushalte kaum an die Betroffenen weitergeleitet werden können (und sollten).

In Tabelle 4 folgt ein Überblick darüber, was die einzelnen Staaten gemäß den vorgestellten Modellen im Vergleich zum derzeit bestehenden System im Haushaltsentwurf 2006 mehr oder weniger bezahlen würden.

7 Wir behandeln in dem Modell auch das Aufkommen aus der Mineralölsteuer wie eine zusätzliche Quelle und die Umleitung der bis dato nationalen Erträge aus diesem Feld gehen nicht in die Berechnungen ein, weil es dazu bisher keine Schätzungen gibt. 
Tabelle 4: Reformmodelle im Vergleich zum Status quo

\begin{tabular}{|c|c|c|c|c|}
\hline & & Modell ,Bundestag“ & $\begin{array}{l}\text { Modell ,Ressourcen- } \\
\text { verbrauch }{ }^{6 *}\end{array}$ & $\begin{array}{l}\text { Modell ,Europa- } \\
\text { mehrwert } * * *\end{array}$ \\
\hline 1. & Belgien & -528 & -2.021 & -488 \\
\hline 2. & Dänemark & -285 & -1.471 & -497 \\
\hline 3. & Deutschland & -1.832 & -14.643 & -3.179 \\
\hline 4. & Estland & -15 & -71 & -8 \\
\hline 5. & Finnland & -282 & -1.182 & -368 \\
\hline 6. & Frankreich & -3.103 & -13.064 & -3.422 \\
\hline 7. & Griechenland & -405 & -1.420 & -216 \\
\hline 8. & Großbritannien & 3.392 & -6.831 & 4.270 \\
\hline 9. & Irland & -270 & -1.053 & -162 \\
\hline 10. & Italien & -2.366 & -10.473 & -3.091 \\
\hline 11. & Lettland & -22 & -93 & -26 \\
\hline 12. & Litauen & -41 & -154 & -6 \\
\hline 13. & Luxemburg & -52 & -194 & -6 \\
\hline 14. & Malta & -9 & -33 & 6 \\
\hline 15. & Niederlande & -263 & -2.987 & -253 \\
\hline 16. & Österreich & -216 & -1.620 & -356 \\
\hline 17. & Polen & -518 & -1.797 & -306 \\
\hline 18. & Portugal & -220 & -1.057 & -8 \\
\hline 19. & Schweden & -364 & -2.004 & -523 \\
\hline 20. & Slowakei & -74 & -283 & -91 \\
\hline 21. & Slowenien & -65 & -220 & -41 \\
\hline 22. & Spanien & -1.565 & -6.618 & -534 \\
\hline 23. & Tschechien & -215 & -734 & -64 \\
\hline 24. & Ungarn & -201 & -662 & -197 \\
\hline 25. & Zypern & -24 & -96 & 23 \\
\hline
\end{tabular}

alle Zahlen in Millionen Euro (für 2006)

* Die projizierten jährlichen Gesamteinnahmen aus den einzelnen Abgabenkategorien verteilen sich nach unseren Schätzungen folgendermaßen: CO2 Zertifikate: 500, Flugmaut: 10.123, Mineralölanteil: 50.617, TOR (90\%): 17.070, BNE: 22.923. Diese Berechnung der Veränderungen nimmt alle Energiemaßnahmen als externe Quellen auf, so dass auch die Beträge der Mineralölsteuer, die aus den nationalen Haushalten abgeführt werden, wie neue Abgaben betrachtet werden. Die nationalen Mindereinnahmen gehen damit nicht in die nationalen Veränderungen ein.

** Die projizierten Gesamteinnahmen aus den Abgaben verteilen sich in etwa folgendermaßen: $1 \%$ der Mehrwertsteuer: 53.262, TOR (90\%): 17.070, BNE: 30.901. Die veränderten Mehrwertsteuer-Anteile fließen in die Berechnung der veränderten nationalen Beiträge ein. 


\section{Neue Spielräume eröffnen}

Das Haushaltssystem steckt in einem Teufelskreis. Der Eigenmittelbeschluss, der allein vom Rat aufgestellt wird, hat gleichzeitig durch die Eigenlogik des , national bargaining ' auf der Ausgabenseite eine Struktur hervorgerufen, die zum weit überwiegenden Teil aus additiven nationalen Ausgabenprogrammen besteht. Wenn man aber die Regierungsperspektive verlässt und die europäischen Bürger fragt, bekommt man schnell den Unmut über diese Ausgabenpolitik zu spüren, weil sie in ihrer Ausrichtung nicht mehr den Bedürfnissen unserer Gesellschaften gerecht wird.

Um den gordischen Knoten durchschlagen zu können muss sich die Überprüfung des Haushalts ab 2008 beiden Seiten mit gleicher Aufmerksamkeit widmen. Positive Ergebnisse können nur dann erreicht werden, wenn der Druck auf eine nachhaltige und zukunftsorientierte Ausgabenpolitik erhöht wird. Die vergangenen Jahre haben uns jedoch gelehrt, dass dies nicht gelingt, solange diese Interessen allein durch die Regierungen und ihren juste-retour-Ansatz definiert werden. Politik auf europäischer Ebene sollte von den Bürgern ausgehen, und aus ihrer Sicht müssen eben nicht nur die Ausgaben auf ihre Bedürfnisse eingehen, sondern auch das Abgabenniveau muss akzeptabel sein. Flankiert durch eine weitgehende Ausgabenreform halten wir das Modell ,Europamehrwert' derzeit für die beste Lösung, weil es Fairness und Transparenz auf der Einnahmeseite einführt. Kernpunkt dieses Modells ist die Verbindung von Konsumausgaben der Bürger mit der Finanzierung der Union.

Unabhängig von der Einführung eines direkten Abgabenbezugs zwischen den Bürgern und Europa lohnt es sich, in der Energie- und Ressourcenpolitik europäischer zu denken und entsprechende Modelle zu entwickeln. Die Überführung bestimmter Abgaben auf die europäische Ebene kann nicht nur zur Stimulierung von Lenkungseffekten genutzt werden, sondern ist oft auch die einzig sinnvolle Lösung für grenzüberschreitende Herausforderungen. $\mathrm{Ob}$ wir diese Steuerungspolitiken auf europäischer Ebene durchführen, muss aber in erster Linie eine energie- und marktpolitische Entscheidung sein. Die Lösung der Eigenmittelproblematik lässt sich schwierig durch die energiepolitische Hintertür erreichen. Darum ist das Modell ,Europamehrwert' für uns die bessere Alternative.

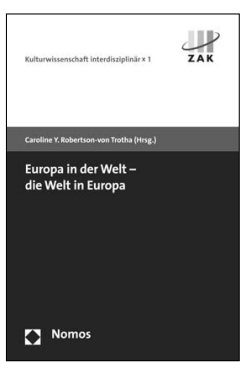

Bitte bestellen Sie bei Ihrer Buchhandlung oder bei: Nomos Verlagsgesellschaft | 76520 Baden-Baden | www.nomos.de

\section{Europa in der Welt - die Welt in Europa}

Herausgegeben von PD Dr. Caroline Y. Robertson-von Trotha, Direktorin des Zentrums für Angewandte Kulturwissenschaft und Studium Generale der Universität Karlsruhe (TH)

2006, 211 S., brosch., 24,- $€$, ISBN 3-8329-1934-1

Vor dem Hintergrund des voranschreitenden Globalisierungsprozesses und der aktuellen Weltlage wird die Frage nach der Identität und der Rolle des modernen Europa in der Welt neu gestellt. In dem Band diskutieren Wissenschaftler und Praktiker die Wertgrundlagen Europas in einer globalisierten Welt.

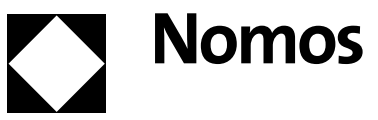

